willigungsunfähigkeit, welche auf diese Weise im Kontext prädiktiver genetischer Untersuchungen zumindest bei Anlegung eines strengen Betrachtungsmaßstabes gar die Regel werden könnte, wodurch die Ratio der Schutzvorschrift in nahezu paternalistisch anmutender Weise überspannt würde.

\section{Fazit}

Im Ergebnis lässt sich festhalten, dass die ohnehin schon mit komplexen ethischen, medizinischen und juristischen Fragestellungen behaftete prädiktive genetische Testung im Zusammenhang mit Minderjährigen eine zusätzliche Problemdimension entfaltet. Bei einwilligungsunfähigen Minderjährigen kommt ihre Vornahme gemäß \$14 Abs. 1 GenDG nur im Hinblick auf solche Erbkrankheiten in Betracht, bei denen Präventions- oder Behandlungsmaßnahmen verfügbar und bereits im jungen Alter indiziert sind, denn ansonsten wäre die Beeinträchtigung des Rechts auf Nichtwissen infolge einer irreversiblen Kenntnis auch kaum zu rechtfertigen. Einwilligungsfähige Minderjährige hingegen sind nach dem Willen des Gesetzgebers unabhängig vom Bestehen solcher medizinischen Handlungsoptionen zu einer selbstbestimmten Entscheidung für oder gegen eine prädiktive Testung berechtigt, im Rahmen derer sie sowohl ihr Recht auf Nichtwissen als auch ihr Recht auf Wissen ausüben können. Obwohl die eigenverantwortliche Bestimmung des Umfangs von Nichtwissen oder eben auch Wissen über relevante und identitätsprägende genetische Daten Ausdruck personeller Autonomie und persönlicher Entfaltung ist ${ }^{32}$, wird das nachvollziehbare und rechtlich anerkannte Interesse an der Erlangung von Gewissheit über die Trägerschaft einer krankheitsassoziierten Mutation in der Praxis missachtet und das Recht auf Wissen durch die restriktive Handhabung dieser Fälle so in bedenklicher Weise beeinträchtigt.

Der Umstand, dass das Gesetz eine bestimmte ärztliche Leistung gesetzlich erlaubt bzw. vorsieht, diese tatsächlich aber zurückhaltend angeboten oder ihre Erbringung gar abgelehnt wird, ist aus anderen, ebenfalls mit ethisch-moralischen Problemen behafteten Konstellationen bekannt ${ }^{33}$. Wenngleich auch die Vornahme prädiktiver genetischer Testungen auf das Vorliegen einer schwerwiegenden und unheilbaren Krankheit bei Minderjährigen durchaus in Konflikt mit dem Gewissen einzelner Mediziner geraten kann, erscheint die pauschale Verweigerung einer Leistungserbringung in diesem Kontext durchaus kritikwürdig und im Ergebnis nicht zu rechtfertigen. Denn die ihr zugrundeliegenden Zweifel, ob der Minderjährige die Folgen des erlangten Wissens einzuschätzen und mit diesem umzugehen vermag, können im Rahmen der Prüfung der individuellen Einwilligungsfähigkeit ausreichend berücksichtigt werden und lösen sich bei lebensnaher Betrachtung wohl auch in den wenigsten Fällen am Tag der Vollendung des 18. Lebensjahres auf.

Die nichtsdestotrotz praktizierte Gleichstellung von Einwilligungsfähigkeit und Volljährigkeit steht in offensichtlichem Widerspruch zu den einfachgesetzlichen Regelungen und hat Pauschalierungen sowie Restriktionen zur Folge, die der Gesetzgeber - wie aus seiner Entscheidung für die Maßgeblichkeit der Einwilligungsfähigkeit hervorgeht - im Zusammenhang mit der Zulässigkeit genetischer Untersuchungen gerade nicht intendiert hat. Gleichwohl leisten die Wertungswidersprüche und Unsicherheiten, welche daraus resultieren, dass einwilligungsfähige Minderjährige lediglich die Kompetenz zur Entscheidung über die Vornahme einer genetischen Testung, nicht aber zum Abschluss des entsprechenden Vertrages innehaben, einen Beitrag zur Entwertung ihres Selbstbestimmungsrechts ${ }^{34}$. Um dieser Personengruppe einen umfassenden Zugang zu prädiktiven genetischen Untersuchungen $\mathrm{zu}$ eröffnen, muss jedenfalls dringend ein gesteigertes Bewusstsein dafür geschaffen werden, dass Volljährigkeit keine Zulässigkeitsvoraussetzung ist. Damit einher geht die grundlegende Anerkenntnis, dass nicht nur dem Recht auf Nichtwissen, sondern auch dem Recht auf Wissen eine hohe Bedeutung zukommt und dieses von Gesetzes wegen dem einwilligungsfähigen Minderjährigen in gleicher Weise wie dem einwilligungsfähigen Volljährigen zusteht.

32) So Meyer, Genetische Untersuchungen zu medizinischen Zwecken und zu Forschungszwecken an nicht einwilligungsfähigen Personen, 2017, S. 50.

33) Exemplarisch zu nennen sind in diesem Zusammenhang etwa Schwangerschaftsabbrüche oder die Beihilfe zum Suizid.

34) Ausführlich zu den zivilrechtlichen Problemfeldern und dem Lösungsvorschlag einer partiellen Geschäftsfähigkeit vgl. Opper/ Fangerau/Braune/Gassner, Prädikative und präsymptomatische Diagnostik spätmanifestierender Erkrankungen. Empfehlungen zur Testung einwilligungsfähiger Minderjähriger, 2019, S. $82 \mathrm{ff}$.

\title{
Die Konsolidierung des Krankenhaussektors - Anpassung der Fusionskontrolle für Krankenhäuser durch die 10. GWB-Novelle
}

\section{Alexander Eufinger}

\section{Einführung und Problemaufriss}

Die Struktur des deutschen Krankenhaussektors ist ein hochpolitisches Thema, welches wellenartig immer wieder die mediale und gesellschaftliche Diskussion zum Gesundheitswesen beherrscht. Aus der jüngeren Vergangenheit sei hier nur die Bertelsmann-Studie aus dem Sommer 2019 genannt, die zu dem - kontrovers diskutierten Ergebnis -

Prof. Dr. iur. Alexander Eufinger,

Technische Hochschule Ostwestfalen-Lippe,

Campusallee 12, 32657 Lemgo, Deutschland gelangte, dass eine starke Verringerung der Klinikanzahl von aktuell knapp 1.400 auf deutlich unter 600 Krankenhäuser, die Qualität der Patientenversorgung verbessern und bestehende Engpässe bei Ärzten und Pflegepersonal mildern würde ${ }^{1}$. Politisch wird diese Debatte oftmals aus

1) BertelsmannStiftung, Zukunftsfähige Krankenhausversorgung, Simulation und Analyse einer Neustrukturierung der Krankenhausversorgung am Beispiel einer Versorgungsregion in NordrheinWestfalen, abrufbar unter https://www.bertelsmann-stiftung.de/ fileadmin/files/BSt/Publikationen/GrauePublikationen/VV_ Bericht_KH-Landschaft_final.pdf. 
Sicht zweier Extrempositionen geführt: Auf der einen Seite der ländliche - oft strukturschwache - Raum, wo Lokalpolitiker ,,ihr“ Krankenhaus ,,vor Ort“ erhalten wollen und auf der anderen Seite der großstädtische Raum, der unter einer vermeintlichen Überversorgung von Krankenhausdienstleistungen leidet ${ }^{2}$. Spätestens seit der COVID19-Pandemie ist das Thema der stationären Versorgungslandschaft in Deutschland aktueller denn je. Vielfach wird die breit aufgestellte Krankenhauslandschaft als ein Grund dafür genannt, warum Deutschland die Pandemie - insbesondere im Vergleich zu anderen europäischen Staaten - bislang so gut gemeistert hat. Besonders die geringe Zahl an Todesfällen wird durch die Existenz eines flächendeckenden Krankenhausnetzes erklärt. Neben medizinökonomischen Überlegungen spielen in diesem Zusammenhang auch wettbewerbliche Überlegungen eine wichtige Rolle. Aus wettbewerbsrechtlicher Sicht hat nunmehr der Regierungsentwurf eines Zehnten Gesetzes zur Änderung des Gesetzes gegen Wettbewerbsbeschränkungen für ein Wettbewerbsrecht 4.0 (sog. GWB-Digitalisierungsgesetz) ${ }^{3}$, welcher am 9.9.2020 vom Bundeskabinett beschlossen wurde, den Fokus der Diskussion auf die Erleichterung von Krankenhausfusionen gelenkt. So sollen nach dem Regierungsentwurf zur 10. GWB-Novelle bestimmte Zusammenschlüsse im Krankenhausbereich von der Fusionskontrolle ausgenommen werden. Zusammenschlussvorhaben von Krankenhäusern würden damit wettbewerbsrechtlich privilegiert werden, wodurch der Gesetzgeber zum Ausdruck bringt, dass er Konzentrationstendenzen auf dem deutschen Gesundheitsmarkt - trotz der Erfahrungen der COVID-19-Pandemie - aus gesundheitspolitischer Sicht nach wie vor als besonders wünschenswert erachtet. Vor diesem Hintergrund soll nachfolgend, neben der Vorstellung der geplanten Bereichsausnahme für Fusionsvorhaben von Krankenhäusern im Entwurf zur 10. GWB-Novelle, untersucht werden, ob derartige Änderungen der wettbewerblichen Zusammenschlusskontrolle für Krankenhäuser tatsächlich gerechtfertigt sind. Vornehmlich geht es hierbei um die Frage, ob bei privilegierten Konzentrationsbestrebungen auf dem Klinikmarkt tatsächlich mit zusammenschlussbedingten Qualitätsvorteilen zu rechnen ist.

\section{Bisheriger rechtlicher Rahmen für Zusammenschlussvorhaben auf dem Krankenhausmarkt}

\section{Anwendbarkeit des GWB auf Krankenhäuer}

Untersagungen von Krankenhausfusionen durch das Bundeskartellamt (BKartA) haben immer wieder die Frage aufgeworfen, ob dass GWB überhaupt auf den Krankenhausmarkt anwendbar ist. Mittlerweile ist vom BGH höchstrichterlich entschieden, dass weder die sozialrechtlichen Regelungen der gesetzlichen Krankenversicherung noch die Bestimmungen des Krankenhausfinanzierungsgesetzes die Anwendbarkeit der Fusionskontrollvorschriften ausschließen ${ }^{4}$. So regelt insbesondere $\$ 69$ SGB V nur die Rechtsbeziehungen der Krankenkassen zu den Krankenhäusern, nicht aber die Rechtsbeziehungen von Krankenhäusern untereinander. Auch die Regelungsbereiche der Fusionskontrolle und der Krankenhausfinanzierung sind unterschiedlich. Nach $₫ 1$ Abs. 1 KHG ist der Zweck der Krankenhausfinanzierung die wirtschaftliche Sicherung der Krankenhäuser, sodass die Leistungserbringer Anspruch auf staatliche Förderung haben, welche anhand der Merkmale Bedarfsgerechtigkeit, Leistungsfähigkeit und Kostengünstigkeit in den jeweiligen Krankenhausplan des Landes aufgenommen worden sind (\$S6, 8 KHG). Zwar wird durch die staatliche Förderung und wirtschaftliche Planung des Krankenhauswesens in erheblichem Maße regulierend auf den Marktzutritt, die Marktbedingungen und die Marktentfaltung der Krankenhäuser Einfluss genommen, jedoch werden die Auswirkungen einer Fusion von Krankenhäusern auf die Marktstruktur im Rahmen der Krankenhausfinanzierung und Krankenhausplanung nicht überprüft ${ }^{5}$. Zusammenschlüssen von Krankenhäusern - als ,,normale“ Unternehmen i.S.d. GWB - unterliegen daher keiner sozial- oder krankenhausrechtlichen Bereichsausnahme, mit der Folge, dass die Fusionskontrolle vollumfänglich anwendbar ist.

\section{Geltungsbereich der Fusionskontrolle}

Neben dem Kartellverbot und dem Verbot des Missbrauchs einer marktbeherrschenden Stellung stellt die Fusionskontrolle die dritte Säule des Kartellrechts dar. Bei der Fusionskontrolle gilt das „One-Stop-Shop“-Prinzip (vgl. Art. 21 Abs. 3 FKVO; $\$ 35$ Abs. 3 GWB). Es gibt nie eine parallele Zuständigkeit von Kommission und BKartA, die EUKommission prüft nur die FKVO wogegen das BKartA nur die $\$ S 35$ ff. GWB prüft. Die deutsche Zusammenschlusskontrolle ist in den $\iint 35 \mathrm{ff}$. GWB geregelt. Zusammenschlüsse von Unternehmen, welche die Aufgreifkriterien der $\int \$ 35,37$ GWB überschreiten, sind grundsätzlich fusionskontrollpflichtig und müssen daher beim BKartA angemeldet werden. Erst nach der Freigabe durch das BKartA oder dem Ablauf bestimmter Wartefristen darf der Zusammenschluss vollzogen werden. Ein Zusammenschluss von zwei Kliniken liegt insbesondere beim Erwerb des ganzen oder eines wesentlichen Teils des Vermögens einer anderen Einrichtung, dem Erwerb der unmittelbaren oder mittelbaren Kontrolle über ein anderes Krankenhaus oder beim Erwerb von Anteilen an einem anderen Krankenhausträger, wenn die Anteile allein oder zusammen mit sonstigen, dem Unternehmen bereits gehörenden Anteilen von $25 \%$ oder $50 \%$ des Kapitals des anderen Unternehmens erreichen, vor. Grundsätzlich hat das BKartA eine solche Krankenhausfusion nach $₫ 36$ Abs. 1 Satz 1 GWB zu untersagen, falls durch diese wirksamer Wettbewerb erheblich behindert würde (sog. SIEC-Test) ${ }^{6}$. Das gesetzliche Regelbeispiel für die Annahme einer erheblichen Behinderung wirksamen Wettbewerbs ist die Entstehung oder Verstärkung einer marktbeherrschenden Stellung.

\section{Marktabgrenzung}

Für das BKartA ist das Regelbeispiel der marktbeherrschenden Stellung nach wie vor das zentrale Kriterium für die Prüfung der Frage, ob eine Krankenhausfusion zu untersagen ist oder nicht. Nach $\int 18$ Abs. 1 GWB ist ein Unternehmen marktbeherrschend, soweit es als Anbieter oder Nachfrager einer bestimmten Art von Waren oder gewerblichen Leistungen auf dem sachlich und räumlich relevanten Markt ohne Wettbewerber ist, keinem wesentlichen Wettbewerb ausgesetzt ist oder eine im Verhältnis $\mathrm{zu}$ seinen Wettbewerbern überragende Marktstellung hat. Entscheidend für die Beurteilung ob zwei sich zusammenschließende Krankenhäuser über eine marktbeherrschende Stellung verfügen werden, ist die sachliche und räumliche

2) Vgl. nur zur Problematik: Lübking, GuP 2019, $175 \mathrm{ff}$

3) BReg., Entwurf eines Gesetzes zur Änderung des Gesetzes gegen Wettbewerbsbeschränkungen für ein fokussiertes, proaktives und digitales Wettbewerbsrecht 4.0 und anderer wettbewerbsrechtlicher Bestimmungen (GWB-Digitalisierungsgesetz); abrufbar unter https://www.bmwi.de/Redaktion/DE/Downloads/Gesetz/ gesetzentwurf-gwb-digitalisierungsgesetz.pdf?_blob=publication File $\& v=6$

4) BGH, NJW-RR 2008, 1426

5) BGH, NJW-RR 2008, 1426, 1427

6) SIEC = Significant Impediment to Effective Competition (erhebliche Behinderung wirksamen Wettbewerbs). 
Abgrenzung des Krankenhausmarktes ${ }^{7}$. Die Abgrenzung des relevanten Marktes - sowohl in sachlicher, als auch in räumlicher Hinsicht - erfolgt grundsätzlich aus Sicht der jeweiligen Marktgegenseite, wobei dies auf Angebotsmärkten die Nachfrager und auf Nachfragemärkten die Anbieter sind. Danach erfasst der sachliche Markt alle Erzeugnisse oder alle - im Gesundheitswesen relevanten - Dienstleistungen, die sich aufgrund ihrer Merkmale und Eigenschaften zur Befriedigung eines gleich bleibenden Bedarfs besonders eignen und nicht oder nur in geringem Maße mit anderen Erzeugnissen oder Dienstleistungen austauschbar sind. An diesem sog. Bedarfsmarktkonzept halten die deutschen Gerichte in ständiger Rechtsprechung fest ${ }^{8}$. Maßgebliche Nachfrager auf dem für die Zusammenschlusskontrolle von Krankenhäusern relevanten Angebotsmarkt - auch im Anwendungsbereich des Sachleistungsprinzips der gesetzlichen Krankenversicherung - sind die Patienten und ihre Nachfrage nach der Leistung ,,akutstationäre Krankenhausbehandlung “9. Dies gilt unabhängig davon, ob Behandlungsleistungen für gesetzlich oder privat versicherte Patienten angeboten werden ${ }^{10}$. Daher sind reine Privatkliniken, Kliniken für Vorsorge und Rehabilitation sowie Alten- und Pflegeheime im Rahmen der Marktabgrenzung nicht zu berücksichtigen ${ }^{11}$. Die vielfach befürwortete weitere Aufteilung des Marktes nach Fachabteilungen lehnt der BGH ab. Jedenfalls bei einer Fusion von Allgemeinkrankenhäusern sei der sachlich relevante Markt nicht nach medizinischen Fachabteilungen abzugrenzen, da die Patienten regelmäßig die üblichen Krankenhausleistungen insgesamt - entsprechend dem so genannten Sortimentsgedanken - als Bündel nachfragten und es zudem typischerweise vielfältige Überschneidungen zwischen den einzelnen Fachabteilungen der Krankenhäuser gebe ${ }^{12}$. In räumlicher Hinsicht umfasst der relevante Markt alle Patienten, die als Abnehmer für das Angebot der am Zusammenschluss beteiligten Krankenhäuser in Betracht kommen und deren wettbewerbliche Handlungsmöglichkeiten durch den Zusammenschluss betroffen und insbesondere beschränkt werden können. Vor diesem Hintergrund bleiben für den Markt akutstationärer Krankenhausdienstleistungen Patienten außer Betracht, welche die Leistungen der am Zusammenschluss beteiligten Krankenhäuser im Hinblick auf die räumliche Entfernung nicht nachfragen ${ }^{13}$. Zu diesem Zweck ermittelt das BKartA die Patientenströme im Hinblick auf die sich unmittelbar zusammenschließenden Krankenhäuser sowie Kliniken aus den benachbarten Gebieten ${ }^{14}$

\section{Erhebliche Behinderung wirksamen Wettbewerbs}

Durch einen Zusammenschluss auf dem sachlich und räumlich abgegrenzten Krankenhausmarkt würde der Wettbewerb erheblich eingeschränkt, falls er die wettbewerblichen Verhaltensspielräume der Nachfrager - in diesem Fall also der Patienten - beschränken würde. Das ist insbesondere dann der Fall, wenn zu erwarten ist, dass Patienten bei einer Verschlechterung der Behandlungsqualität des aufgesuchten Krankenhauses auf ein Krankenhaus außerhalb des als räumlich relevant betrachteten Markts ausweichen ${ }^{15}$. Die Vermutung, dass insbesondere ein marktbeherrschendes Krankenhaus die Behandlungsqualität zulasten der Patienten reduzieren könnte, ist daher oftmals der Grund für die Untersagung eines geplanten Zusammenschlusses von Krankenhäusern ${ }^{16}$. Auch die Bundesregierung stellt in ihrer Begründung zum Entwurf zur 10. GWB-Novelle fest, dass sich nach empirischen Erkenntnissen das Fehlen von wettbewerblichen Anreizen für Patienten negativ auswirkt ${ }^{17}$. So sind infolge des sinkenden Wettbewerbsdrucks Einbußen der Behandlungsqualität zu befürchten, ohne dass dies durch Größen- oder Spezialisierungseffekte der fusionierenden Kliniken hinreichend kompensiert wird. Andererseits können den negativen wettbewerblichen Auswirkungen eines
Zusammenschlusses Effizienzgewinne entgegenstehen. Dabei sind nur solche Effizienzvorteile für die wettbewerbliche Beurteilung relevant, die eine unmittelbare Folge des Zusammenschlussvorhabens sind und nicht in ähnlichem Umfang durch weniger wettbewerbsschädliche Alternativen erzielt werden können ${ }^{18}$. Derartige Effizienzgewinne sind regelmäßig auf Größenvorteile (Synergieeffekte) zurückzuführen, die sich wiederum auf Kosten und Qualität auswirken. Gleichwohl ist auf dem Krankenhaussektor eine Besonderheit $\mathrm{zu}$ beachten: Aufgrund des fixierten Preises je Behandlungsfall werden sich die Wirkungen von solchen Größenvorteilen primär auf die Angebotsqualität und nicht - wie in anderen Branchen üblich - auf den Preis niederschlagen ${ }^{19}$. Gleichwohl ist aus ökonomischer Sicht eine Mindestgröße eines Krankenhauses lediglich eine notwendige, aber nicht hinreichende Bedingung für eine qualitativ hochwertige und wirtschaftliche Versorgung ${ }^{20}$. Vielmehr ist regelmäßig mit zusammenschlussbedingten Qualitätseinbußen zu Lasten der Patienten zu rechnen. Im Wettbewerb stehende Krankenhausträger haben den Anreiz, medizinische, technische und organisatorische Innovationen einzuführen, um ihre Patienten qualitativ besser und effizienter $\mathrm{zu}$ versorgen, und sich an Veränderungen der Rahmenbedingungen anzupassen. Daher ist gewöhnlicherweise zu erwarten, dass das wettbewerblich orientierte Verhalten von Kliniken ohne einen Zusammenschluss fortdauern wird ${ }^{21}$. Daher verwundert es nicht, dass die Möglichkeit der Anerkennung von Effizienzvorteilen bei Krankenhausfusionen eher verneint wird.

\section{Geplante Änderungen im Fusionskontrollverfahren für Krankenhäuser}

Nach den Plänen der Bundesregierung (vgl. \$186 Abs. 9 GWB-E) soll die Fusionskontrolle ( $\$ \$ 35$ bis 41 GWB) keine Anwendung auf Zusammenschlussvorhaben im Krankenhausbereich finden, soweit der Zusammenschluss eine standortübergreifende Konzentration von mehreren Krankenhäusern oder einzelnen Fachrichtungen mehrerer Krankenhäuser zum Gegenstand hat, dem Zusammenschluss keine anderen wettbewerbsrechtlichen Vorschriften entgegenstehen und dies das Land bei Antragstellung nach \14 Abs. 2 Nr. 3 lit. a) der Krankenhausstrukturfonds-Verordnung (KHSFV) bestätigt hat, das Vorliegen der weiteren Voraussetzungen für eine Förderung nach $\$ 12 \mathrm{a} A \mathrm{~b} .1$

7) Bold, in: Huster/Kaltenborn, Krankenhausrecht, 2. Aufl. 2017, \11, Rdnr. 28

8) Vgl. statt aller BGH, NZS 2008, 653.

9) Vgl. BKartA, Beschl. v. 27.5.2013, B3-17/13

10) BGH, NZS 2008,653

11) Bold, in: Huster/Kaltenborn, Krankenhausrecht, 2. Aufl. 2017, \11, Rdnr. 31

12) BGH, NJW-RR 2008, 1426, 1430; hierzu auch Kirchhoff, GRUR 2009, 284, 287

13) BGH, NZS 2008,653

14) Bold, in: Huster/Kaltenborn, Krankenhausrecht, 2. Aufl. 2017, \11, Rdnr. 33; kritisch hierzu Augursky/Jendges, Strukturanpassungen im Krankenhausbereich: Notwendige Veränderungen bei der Fusionskontrolle, RWI Positionen 73, 2019.

15) BGH, NJW-RR 2008, 1426, 1431, 1432.

16) Monopolkommission, Hauptgutachten XXIII: Wettbewerb 2020, S. 65.

17) BReg., Entwurf eines GWB-Digitalisierungsgesetz, S. 171; unter Verweis auf Schmid, Marktkonzentration im Krankenhaussektor, 2018

18) BKartA, Beschl. v. 14.5.2014 - B3-135/13 -, Klinikum Esslingen/Kreiskliniken Esslingen.

19) Monopolkommission, Hauptgutachten XXIII: Wettbewerb 2020, S. 65.

20) BReg., Entwurf eines GWB-Digitalisierungsgesetz, S. 171.

21) BKartA, Beschl. v. 14.5.2014 - B3-135/13 -, Klinikum Esslingen/Kreiskliniken Esslingen. 
S. 4 des Krankenhausfinanzierungsgesetzes in Verbindung mit $₫ 11$ Abs. 1 Nr. 2 KHSFV in einem Auszahlungsbescheid nach $\$ 15$ der Krankenhausstrukturfonds-Verordnung festgestellt wurde und der Zusammenschluss bis zum 31. 12.2025 vollzogen wird.

Liegt also eine Fusion von zwei oder mehreren Krankenhäusern bzw. einzelnen Fachrichtungen verschiedener Kliniken vor, die eine standortübergreifende Wirkung entfaltet, kann ein derartiges Vorhaben nach $\$ 11$ Abs. 1 Nr. 2 KHSFV aus Mitteln des Strukturfonds gefördert werden. Voraussetzung für die Förderung nach $\$ 12 \mathrm{a}$ KHG i.V. mit $§ 11$ Abs. 1 Nr. 2 KHSFV ist, dass dieses Vorhaben wettbewerbsrechtlich zulässig ist. Neben klassischen Zusammenschlüssen von Krankenhäusern sind auch Vorhaben zur Bildung von Zentren zur Behandlung von seltenen, komplexen oder schwerwiegenden Erkrankungen an Hochschulkliniken, Vorhaben zur Bildung von Krankenhausverbünden, Vorhaben zur Bildung integrierter Notfallstrukturen und telemedizinischer Netzwerkstrukturen sowie Vorhaben zur Verbesserung der informationstechnischen Sicherheit der Krankenhäuser förderungswürdig, vorausgesetzt diese Formen der Zusammenarbeit sind wettbewerbsrechtlich zulässig. Mit der geplanten Bereichsausnahme nach \$186 Abs. 9 GWB-E beabsichtigt der Gesetzgeber nunmehr Zusammenschlussvorhaben sowie bestimmte Vorhaben der Kooperation, welche als gesundheitspolitisch besonders wünschenswert und damit als förderungswürdig gemäß dem Krankenhausfinanzierungsgesetz gelten, als per se wettbewerbsrechtlich zulässig zu erachten. Auch wenn man dem Gesetzgeber keine tautologischen Neigungen unterstellen möchte, wird doch bei Inkrafttreten der 10. GWB-Novelle die allgemein gültige Aussage gelten müssen: Ein nach $₫ 12 \mathrm{a}$ KHG i.V. mit $\$ 11$ Abs. 1 Nr. 2 KHSFV i. V. mit $\$ 186$ Abs. 9 GWB-E als gesundheitspolitisch besonders wünschenswert betrachtetes Zusammenschlussvorhaben, mit dem die gesundheitspolitischen Ziele der Spezialisierung und Zentrenbildung verfolgt werden, gilt stets als wettbewerbsrechtlich privilegiert. Eine eigenständige fusionskontrollrechtliche Prüfung hat daher zu unterbleiben. Die Bundesregierung begründet diese Tautologie mit den Argumenten, dass mit der Anknüpfung an die konkrete Förderung die Rechtssicherheit erhöht werde, wettbewerbliche Risiken reduziert und die Steuerungsmöglichkeiten der Länder gewahrt wer$\mathrm{den}^{22}$. Folgerichtig ist die Freistellung eines Zusammenschlusses von Krankenhäusern von der Fusionskontrolle bis zum 31.12.2025 befristet, entspricht dieser Zeitraum doch der in $\$ 12$ a KHG vorgesehenen Laufzeit des Strukturfonds zzgl. einer angemessenen Zeit zur Umsetzung der bewilligten Vorhaben. Diese befristete Bereichsausnahme für Krankenhausfusionen erachtet die Bunderegierung zudem als angemessen, da eventuelle, im Einzelfall mögliche, wettbewerbsrechtliche Bedenken für eine begrenzte Zeit insoweit hinter das insbesondere durch den Strukturfonds verfolgte Konsolidierungsziel zurückzutreten hätten ${ }^{23}$. Diese Ausführungen im Gesetzentwurf der Bundesregierung hinterlassen beim geneigten Leser gleichwohl einen faden Beigeschmack, kann man sich doch des Eindrucks nicht erwehren, dass zur effizienzsteigernden Konsolidierung und ggfs. Spezialisierung deutscher Krankenhäuser wettbewerbsrechtliche Grundprinzipien über Bord geworfen werden. Getreu dem Motto: Der Zweck heiligt (fast) alle Mittel.

\section{Struktur des Krankenhaussektors und Notwendigkeit einer Bereichsausnahme im GWB}

\section{Struktur des Krankenhaussektors und Anpassungsbedarf}

Nach den Angaben des Statistischen Bundesamts existierten im Jahr 2018 insgesamt 1.925 (2017: 1. 942) Krankenhäuser in Deutschland, welche rund 498.200 Betten für die stationäre Versorgung der Bevölkerung zur Verfügung stellten.
Während die Zahl der Häuser um 0,9 Prozent sank, blieb das Bettenangebot gegenüber dem Vorjahr nahezu unverändert (ein Plus von 0,2 Prozent). Die Bettenauslastung lag bei 77,1 Prozent ${ }^{24}$. Im direkten Vergleich mit den anderen EU-Staaten weist die Bundesrepublik damit eine nahezu einmalig hohe Anzahl von über 800 Krankenhausbetten je 100.000 Einwohner aus ${ }^{25}$. Insbesondere der Vergleich mit dem Vereinigten Königreich (250 Krankenhausbetten je 100.000 Einwohner) und skandinavischen Ländern wie Schweden (220 Krankenhausbetten je 100.000 Einwohner) suggeriert eine Überversorgung der Bevölkerung. Problematisch ist in diesem Zusammenhang, dass sich diese hohen Bettenkapazitäten auf viele kleine Krankenhäuser verteilen. Nahezu 65 Prozent aller Allgemeinkrankenhäuser verfügen über weniger als 300 Betten $^{26}$. Viele dieser kleinen Kliniken erreichen daher nicht die gesetzlich vorgeschriebenen Mindestfallzahlen. So dürfen in deutschen Kliniken einige wenige komplexe Operationen und Behandlungen nur dann durchgeführt werden, wenn dort Ärzte und ihre Teams ein Mindestmaß an Erfahrung haben. Dabei handelt es sich ausnahmslos um planbare Eingriffe, bei denen ein Zusammenhang zwischen der Behandlungsqualität und der Fallzahl pro Klinik nachgewiesen wurde. Hintergrund ist, dass wissenschaftlich ein kausaler Zusammenhang zwischen der Höhe der Fallzahlen in einer Klinik und der dortigen Behandlungsqualität belegt ist: Je mehr Patienten mit einer bestimmten Krankheit in einer Klinik behandelt werden, desto weniger Komplikationen und Todesfälle treten auf ${ }^{27}$. Basierend auf diesem Ergebnis verfolgt der Gesetzgeber das Ziel, größere und spezialisierte Versorgungszentren zu bilden, um die Behandlungsqualität und Patientensicherheit $\mathrm{zu}$ erhöhen ${ }^{28}$. Konzentrationsbestrebungen fußen zudem auf dem Gedanken, dass dem ärztlichen und pflegerischen Fachkräftemangel durch eine Verteilung der vorhandenen Personalressourcen auf weniger Kliniken begegnet werden könnte. Argumentiert wird, dass die hohe Krankenhausdichte nur ein vermeintlicher Vorteil sei, gehe diese doch mit Qualitätsmängeln - insbesondere in der Personalausstattung - einher. Auf einem konsolidierten Klinikmarkt könnte das Personal vielmehr im Sinne einer höheren Versorgungsqualität eingesetzt werden ${ }^{29}$. Schließlich ist eines der Hauptargumente für die hohe Dichte an - insbesondere kleinen - Krankenhäusern, dass diese zur flächendeckenden Notfallversorgung der Bevölkerung erforderlich seien. Eine Studie des Leibniz-Instituts für Wirtschaftsforschung im Auftrag der Kassenärztlichen Bundesvereinigung kam jedoch zu dem Ergebnis, dass eine flächendeckende Notfallversorgung mit deutlich weniger Teilnehmern möglich und sinnvoll sei. Aus wirtschaftlichen und versorgungstechnischen Gesichtspunkten sei es nicht effizient, dass alle momentan an der Notfallversorgung teilnehmenden Kliniken diesen Status beibehalten, bzw. durch eine Notfallpraxis der KV ergänzt werden ${ }^{30}$. Im Ergebnis lässt sich feststellen, dass in der Bundesrepublik tendenziell zu viele

22) BReg., Entwurf eines GWB-Digitalisierungsgesetz, S. 171

23) BReg., Entwurf eines GWB-Digitalisierungsgesetz, S. 171.

24) Statistisches Bundesamt, Fachserie 12 Reihe 6.1.1, S. 22.

25) OECD, Health at a Glance 2019: OECD Indicators, Paris 2019, S. 195.

26) Monopolkommission, Hauptgutachten XXIII: Wettbewerb 2020, S. 58.

27) Science Media Center Germany, Mindestmengen im Krankenhaus - Bilanz und Neustart, Endbericht, 2019.

28) So explizit die BReg., Entwurf eines GWB-Digitalisierungsgesetz, S. 170.

29) Monopolkommission, Hauptgutachten XXIII: Wettbewerb 2020, S. 59; so auch das Ergebnis der Bertelsmann-Studie aus dem Jahr 2019.

30) RWI, Notfallversorgung in Deutschland, Projektbericht im Auftrag der Kassenärztlichen Bundesvereinigung, 2018, S. 69. 
kleine Krankenhäuser mit einem zu geringen Grad an medizinischer Spezialisierung vorgehalten werden ${ }^{31}$. Die Zentralisierung und Konzentration von Krankenhäusern wird daher als gesundheitspolitisch wünschenswert erachtet ${ }^{32}$.

\section{Notwendigkeit einer Bereichsausnahme im GWB?}

Schließen sich zwei Krankenhäuser zusammen, bietet dies dem alleinigen Krankenhausbetreiber die Möglichkeit, die Häuser neu zu organisieren und die gesundheitspolitisch gewünschten Spezialisierungs- und Zentralisierungsziele direkt umzusetzen ${ }^{33}$. Fusionen auf dem Krankenhausmarkt sind damit ein effektives und - vor allem - schnell wirkendes Instrument, um die Konsolidierung des deutschen Klinikmarktes $\mathrm{zu}$ erreichen. Aus gesundheitspolitischer Perspektive hat dieses Instrument nur einen entscheidenden Nachteil: Ein solcher Zusammenschluss unterliegt wie bereits ausgeführt - regelmäßig der Fusionskontrolle nach den $\$ \$ 35 \mathrm{ff}$. GWB und das BKartA hat in der Vergangenheit wenig Motivation gezeigt, die besondere Situation des Krankenhausmarktes zu berücksichtigen ${ }^{34}$. Bislang berücksichtigt das BKartA vornehmlich die „Diversion Ratio“" (sog. Umlenkungsquote) zwischen den Zusammenschlussbeteiligten. Hierbei wird ermittelt, wie niedergelassene Ärzte ihre Einweisungsempfehlungen bei Wegfall eines Krankenhauses hypothetisch ändern würden. Eine hohe Umlenkungsquote zwischen zwei fusionierenden Kliniken indiziert regelmäßig ein enges Wettbewerbsverhältnis zwischen den Zusammenschlussbeteiligten, wodurch sich der Wettbewerb im Falle der Fusion weiter verringern wür$\mathrm{de}^{35}$. Diese Praxis des BKartA hat im Ergebnis zur Folge, dass eine besondere wettbewerbliche Nähe der Zusammenschlussbeteiligten häufig zu einer erheblichen Behinderung wirksamen Wettbewerbs führen wird, da den niedergelassenen Ärzten ein Leistungserbringer für ihre Einweisungsempfehlungen wegfallen würde. Im Umkehrschluss gilt jedoch auch: Ist durch einen geplanten Zusammenschluss auch keine Veränderung des Einweisungsverhaltens der Ärzte zu Lasten der Wettbewerber zu erwarten - die den Verhaltensspielraum der am Zusammenschluss Beteiligten erweitern könnten - wird der Wettbewerb auch nicht (erheblich) gemindert ${ }^{36}$.

Obgleich es aus gesundheitspolitischer Sicht angezeigt ist, hat das BKartA bislang die Auswirkungen eines $\mathrm{Zu}-$ sammenschlusses auf die medizinische Versorgungsqualität und die mit der Fusion einhergehenden Anderungen bei der Erreichbarkeit durch die Patienten kaum in den Fokus genommen. So machten etwa die Beteiligten im Verfahren Klinikum Esslingen/Kreiskliniken Esslingen darauf aufmerksam, dass der intendierte Zusammenschluss der Krankenhäuser zu einer verbesserten Qualität der Patientenversorgung führe, da komplexe oder weniger häufige Erkrankungen an einem Standort konzentriert würden, während die ,Grund- und Regelversorgung“ in der Fläche erhalten bleibe ${ }^{37}$. Zudem sichere der Zusammenschluss in verschiedenen Bereichen Fallzahlen, die im Bereich der Mindestmengendiskussion eine sehr gute Ausgangslage biete. Das BKartA sah diese Vorteile als nicht gegeben bzw. nicht fusionsspezifisch an. So müsse ein Zusammenschluss zu nachprüfbaren zusammenschlussbedingten Vorteilen für die Patienten als Verbrauchern führen, welche den Wegfall des engsten Wettbewerbers und somit der für die Patienten wichtigsten Behandlungsalternative kompensieren. Im streitgegenständlichen Verfahren seien die fachlichen Vorteile aus der Bündelung von Fachabteilungen aus Sicht des Amtes auch ohne den Zusammenschluss erreich$\mathrm{bar}^{38}$. Zudem bezweifelt das BKartA regelmäßig, dass mögliche zusammenschlussbedingte Effizienzvorteile aus dem geplanten Abbau von Doppelstrukturen tatsächlich den $\mathrm{Pa}-$ tienten zugutekommen werden. Das Interesse für die fusionierten Krankenhäuser, Effizienzvorteile an die Patienten weiterzugeben, hängt davon $a b$, ob durch die im Markt verbleibenden Unternehmen oder von einem potenziellen Markteintritt Wettbewerbsdruck ausgeht. Bei einer starken Marktstellung der Krankenhäuser nach einem Zusammenschluss - wovon häufig auszugehen sein wird - geht die Kartellbehörde davon aus, dass mit einem Weiterreichen der Effizienzen an die Patienten nicht zu rechnen sei ${ }^{39}$. Die bisherige Entscheidungspraxis des BKartA zeigt, dass die Kartellbehörde Zusammenschlussvorhaben im Krankenhausmarkt durchaus kritisch gegenübersteht und die gleichen Prüfkriterien wie bei Fusionen von sonstigen Unternehmen anlegt. Gleichwohl lässt eine Entscheidung des BKartA aus der jüngeren Vergangenheit erkennen, dass die Kartellbehörde leistungs- und qualitätsspezifische Aspekte stärker in ihr Prüfprogramm einfließen lässt ${ }^{40}$. Da diese Aspekte wettbewerbsökonomisch schwierig zu bestimmen sind, greift das BKartA auch hier auf die Ergebnisse der Befragung der niedergelassenen Ärzte in der jeweiligen Region zurück. So geben die niedergelassenen Ärzte ihren Patienten üblicherweise Ratschläge zur Krankenhauswahl und schätzen die Bedeutung dieser Empfehlungen für die Auswahlentscheidung ihrer Patienten berechtigterweise als sehr hoch ein. Das Beratungs- und Empfehlungsverhalten der niedergelassenen Ärzte wiederum wird maßgeblich durch ihre Wahrnehmungen zu Qualität und Leistungsspektrum der Krankenhäuser in ihrer Region beeinflusst ${ }^{41}$. Auf eine sinkende Qualität oder ein geringeres Leistungsspektrum reagieren die niedergelassenen Ärzte überwiegend mit weniger Empfehlungen. Umgekehrt reagieren die Ärzte auf eine von ihnen beobachtete gesteigerte Behandlungsqualität auch mit mehr Empfehlungen, sodass sich wettbewerbliche Vorstöße von Konkurrenten der $\mathrm{Zu}$ sammenschlussbeteiligten auch in höheren Fallzahlen niederschlagen werden ${ }^{42}$.

Die bisherige behördliche Spruchpraxis steht damit im Widerspruch zu den Bestrebungen des Gesetzgebers, der durch die Novellierung des GWB gesundheitspolitisch bezweckte Strukturveränderungen beschleunigen möchte und in diesem Zusammenhang unumwunden einräumt, dass wettbewerbsrechtliche Bedenken hinter diesem Konsolidierungsziel zurückzutreten haben ${ }^{43}$. Eine kartellrechtliche Bereichsausnahme im Krankenhaussektor hätte damit zur Folge, dass hinter den planerischen Interessen im

31) Monopolkommission, Hauptgutachten XXIII: Wettbewerb 2020, S. 60; Schmid, Marktkonzentration im Krankenhaussektor, 2018, S. 23.

32) BReg., Entwurf eines GWB-Digitalisierungsgesetz, S. 170.

33) Monopolkommission, Hauptgutachten XXIII: Wettbewerb 2020, S. 60.

34) Vgl. statt aller: Augursky/Jendges, Strukturanpassungen im Krankenhausbereich: Notwendige Veränderungen bei der Fusionskontrolle, RWI Positionen 73, 2019.

35) BKartA, Beschl. v. 17. 12.2018 - B3-122/18 -, Cellitinnen Köln.

36) BKartA, Beschl. v. 30.7.2020 - B3-33/20 -, MALTESER-DIAKO KLINIKUM Flensburg.

37) BKartA, Beschl. v. 14. 5.2014 - B3-135/13 -, Klinikum Esslingen/Kreiskliniken Esslingen.

38) BKartA, Beschl. v. 14.5.2014 - B3-135/13 -, Klinikum Esslingen/Kreiskliniken Esslingen.

39) BKartA, Beschl. v. 14.5.2014 - B3-135/13 -, Klinikum Esslingen/Kreiskliniken Esslingen.

40) Vgl. BKartA, Beschl. v. 30.7.2020 - B3-33/20 -, MALTESERDIAKO KLINIKUM Flensburg.

41) Sicherlich mag es in diesem Zusammenhang auch schwarze Schafe geben, deren Zuweisungsverhalten mehr durch monetäre Anreize, als durch die qualitativen Vorteile eines Krankenhauses beeinflusst wird; wobei derartige Praktiken - insbesondere unter Berücksichtigung der \$S299a und 299b StGB - eher die Ausnahme sind.

42) BKartA, Beschl. v. 30. 7.2020 - B3-33/20 -, MALTESER-DIAKO KLINIKUM Flensburg.

43) BReg., Entwurf eines GWB-Digitalisierungsgesetz, S. 171. 
Sinne einer Konzentration von mehreren Krankenhäusern - ebenso wie eine Zusammenarbeit einzelner Fachrichtungen mehrerer Krankenhäuser - sämtliche sonstigen wettbewerbsrechtlichen Überlegungen zurückzutreten haben. Insbesondere die qualitätssichernden Effekte eines höheren Wettbewerbsdrucks durch eine Vielzahl konkurrierender Krankenhäuser würden gänzlich außer Betracht gelassen. Aus wettbewerbsrechtlicher Sicht ist eine kartellrechtliche Bereichsausnahme für den Krankenhaussektor daher äußerst kritisch zu würdigen: So werden wettbewerbliche Grundprinzipien auf dem Altar der gesundheitspolitisch gewünschten Konsolidierung des Krankenhausmarktes geopfert $^{44}$. Dass selbst der Bundesregierung gewisse Zweifel an der geplanten Änderung des GWB kommen, demonstrieren die Ausführungen in ihrer Gesetzesbegründung: Apodiktisch wird konstatiert, dass trotz der mit $\$ 186$ Abs. 9 GWB-E geschaffenen Ausnahmeregelung der Wettbewerb zum Schutz einer qualitativ hochwertigen Versorgung und die Trägervielfalt im Krankenhaussektor grundsätzlich aufrechterhalten werden soll ${ }^{45}$. Nicht unbegründet sind daher auch die Bedenken der Bundesregierung, wonach mit einer weitgehenden Ausnahme von der Fusionskontrolle insbesondere Finanzinvestoren ein uneingeschränkter Zugriff auf die deutsche Krankenhauslandschaft eröffnet werde, da Verschiebungen der Marktanteile zugunsten weniger großer Konzerne keine kartellrechtlichen Hürden mehr entgegenstünden ${ }^{46}$. Besonders bemerkenswert ist, dass der Sachverständigenrat der Bundesregierung zur Begutachtung der Entwicklung im Gesundheitswesen eine nahezu gleichlautende Position bereits in seinem Gutachten aus dem Jahr 2007 vertrat. So sollte vor dem Hintergrund der Bestrebungen, auch im Gesundheitssektor zu einer stärker wettbewerblich orientierten Struktur zu gelangen, schon damals dafür Sorge getragen werden, dass keine marktbeherrschenden Stellungen auf Seite der Leistungserbringer entstehen ${ }^{47}$. Begründet wurde dies unter anderem mit Erkenntnissen aus US-amerikanischen Studien, nach denen Krankenhausfusionen mit deutlichen Preisanstiegen einhergehen können. Aus diesem Grund sei eine ,konsequente Anwendung " der Fusionskontrolle im Krankenhaussektor zu befürworten $^{48}$. Eine Marktstruktur, die einen Qualitätswettbewerb ermöglicht, gibt den Krankenhäusern einen ständigen Anreiz zu Qualitätsverbesserungen, sodass die Zusammenschlusskontrolle - welche diesen Qualitätswettbewerb schützt - letztlich einen wesentlichen Beitrag zur Verwirklichung gesundheitspolitischer Ziele leistet ${ }^{49}$.

\section{Fazit}

Die im Zuge der 10. GWB-Novelle geplante kartellrechtliche Bereichsausnahme im Krankenhaussektor steht im
Gegensatz zum tradierten System der Zusammenschlusskontrolle. Das bislang bewährte Zusammenspiel sozial- und gesundheitspolitischer sowie wettbewerblicher Steuerungsmechanismen wird hierdurch zu Lasten des Wettbewerbs - zumindest bis zum 31.12.2025 - aufgegeben. Zudem wird durch die geplante Regelung, wonach für eine fusionsrechtliche Privilegierung eine Förderung nach $₫ 12 \mathrm{a}$ Ab. $1 \mathrm{~S} .4$ des Krankenhausfinanzierungsgesetzes in Verbindung mit $\$ 11$ Abs. 1 Nr. 2 KHSFV erforderlich ist, die Trennung zwischen krankenhaus- und wettbewerbsrechtlichen Vorgaben aufgegeben, wird doch die kartellrechtliche Bereichsausnahme auf solche Vorhaben begrenzt, die aus Mitteln des Strukturfonds im Interesse einer hohen Versorgungsqualität gefördert werden. Zwar kann die gesundheitspolitisch gewünschte Konsolidierung des Krankenhausmarktes am einfachsten über Zusammenschlüsse der Leistungserbinger erreicht werden, dennoch dürften infolge des sinkenden Wettbewerbsdrucks EinbuBen bei der Behandlungsqualität zu befürchten sein. Um solche fusionsbedingten Qualitätseinbußen zu Lasten der Patienten zu vermeiden, sollte die Bundesregierung von ihrem Gesetzesvorhaben absehen. Anstatt den Krankenhaussektor der kartellrechtlichen Zusammenschlusskontrolle durch eine Novellierung des GWB zu entziehen, sollte der Gesetzgeber durch eine Ergänzung im GWB das BKartA verpflichten, bei seiner Entscheidungspraxis die Besonderheiten des Klinikmarktes stärker als bisher $\mathrm{zu}$ berücksichtigen ${ }^{50}$. Insbesondere eine Abwägung der aus der Fusion entspringenden Qualitätsvorteile mit dem Verlust des wettbewerblichen Drucks und den damit einhergehender Qualitätseinbußen erscheint sachgerecht. In diesem Zusammenhang kann besonders die zusammenschlussbedingte Verknüpfung von Fachabteilungen zu einer konzentrierteren Behandlung komplexer Krankheitsbilder und einer Erhöhung von Fallzahlen führen. Diese Effekte würden unmittelbar den Patienten zugute kommen und sollten daher im Rahmen des SIEC-Test berücksichtigt werden ${ }^{51}$.

44) So auch Monopolkommission, Hauptgutachten XXIII: Wettbewerb 2020, S. 68.

45) BReg., Entwurf eines GWB-Digitalisierungsgesetz, S. 171.

46) BReg., Entwurf eines GWB-Digitalisierungsgesetz, S. 171.

47) Sachverständigenrat zur Begutachtung der Entwicklung im Gesundheitswesen, Gutachten 2007, S. 192.

48) Sachverständigenrat zur Begutachtung der Entwicklung im Gesundheitswesen, Gutachten 2007, S. 192.

49) Kirchhoff, GRUR 2009, 284, 288.

50) Monopolkommission, Hauptgutachten XXIII: Wettbewerb 2020, S. 68.

51) Siehe Abschnitt IV. 1. 